\title{
Two-dimensional real symmetric spaces with maximal projection constant
}

\author{
by Bruce L. Chalmers (Riverside, CA) and \\ GrZEGORZ LEWICKI (Kraków)
}

\begin{abstract}
Let $V$ be a two-dimensional real symmetric space with unit ball having $8 n$ extreme points. Let $\lambda(V)$ denote the absolute projection constant of $V$. We show that $\lambda(V) \leq \lambda\left(V_{n}\right)$ where $V_{n}$ is the space whose ball is a regular $8 n$-polygon. Also we reprove a result of [1] and [5] which states that $4 / \pi=\lambda\left(l_{2}^{(2)}\right) \geq \lambda(V)$ for any two-dimensional real symmetric space $V$.
\end{abstract}

Introduction. Let $X$ be a normed space and let $V$ be a linear subspace of $X$. Denote by $\mathcal{P}(X, V)$ the set of all projections from $X$ onto $V$, i.e., the set of all continuous extensions of id $: \mathrm{V} \rightarrow \mathrm{V}$ onto $X$. Let

$$
\begin{aligned}
\lambda(V, X) & =\inf \{\|P\|: P \in \mathcal{P}(X, V)\}, \\
\lambda(V) & =\sup \{\lambda(V, X): V \subset X\} .
\end{aligned}
$$

We call $\lambda(V, X)$ the relative projection constant of $V$ in $X$ and $\lambda(V)$ the $a b$ solute projection constant of $V$. A projection $P \in \mathcal{P}(X, V)$ is called minimal if $\|P\|=\lambda(V, X)$. Observe that the problem of finding minimal projections is related to the Hahn-Banach theorem, since we are looking for a minimal norm extension of the identity operator on $V$.

In this note we show that, for any two-dimensional real symmetric space $V$ with a polygonal unit ball having $8 n$ extreme points,

$$
\lambda(V) \leq \lambda\left(V_{n}\right),
$$

where $V_{n}$ is the space whose unit ball is regular $8 n$-polygon. As an application of (1.3) we reprove a result of [1] and [5] which states that

$$
4 / \pi=\lambda\left(l_{2}^{(2)}\right) \geq \lambda(V)
$$

for any two-dimensional real symmetric space $V$.

2000 Mathematics Subject Classification: 41A35, 41A65, 47A30.

Key words and phrases: absolute projection constant, minimal projection, symmetric spaces. 
Now we introduce some notation which will be of use later. By $S_{V}$ we will denote the unit sphere in a normed space $V$. The symbol ext $\left(S_{V}\right)$ will stand for the set of all extreme points of $S_{V}$. Note that if $V$ is a $k$-dimensional subspace of $l_{1}^{(n)}$ then each $P \in \mathcal{P}\left(l_{1}^{(n)}, V\right)$ has the form

$$
P x=\sum_{i=1}^{k} u^{i}(x) v^{i},
$$

where $v^{1}, \ldots, v^{k}$ is a fixed basis of $V$ and $u^{1}, \ldots, u^{k} \in l_{\infty}^{(n)}$ satisfy

$$
u^{j}\left(v^{i}\right)=\sum_{l=1}^{n} u_{l}^{j} v_{l}^{i}=\delta_{i j} .
$$

A point $x \in X$ is called a norming point for $f \in X^{*}$ if

$$
x \in S_{X} \quad \text { and } \quad f(x)=\|f\| .
$$

DeFinition 1.1. Let $V$ be a finite-dimensional Banach space. It is called symmetric if there exists a basis $v^{1}, \ldots, v^{k}$ in $V$ such that

$$
\left\|\sum_{i=1}^{k}\left|\alpha_{i}\right| v^{i}\right\|=\left\|\sum_{i=1}^{k} \alpha_{\pi(i)} v^{i}\right\|
$$

for any $\alpha_{1}, \ldots, \alpha_{k} \in \mathbb{R}$ and any permutation $\pi$ of the indices.

Now let $P=\sum_{i=1}^{k} u^{i}(\cdot) v^{i} \in \mathcal{P}\left(l_{1}^{n}, V\right)$. Define

$$
\operatorname{crit}(P)=\left\{j \in\{1, \ldots, n\}:\left\|P e_{j}\right\|=\|P\|\right\}
$$

and for $j=1, \ldots, n$,

$$
V_{j}=\left(v_{j}^{1}, \ldots, v_{j}^{k}\right), \quad U_{j}=\left(u_{j}^{1}, \ldots, u_{j}^{k}\right) .
$$

TheOrem 1.2 [2, Th. 3, p. 294]. Let $P=\sum_{i=1}^{k} u^{i}(\cdot) v^{i} \in \mathcal{P}\left(l_{1}^{(n)}, V\right)$. Then $P$ is minimal if and only if there exists a nonzero $k \times k$ matrix $M$ such that for every $j \in \operatorname{crit}(P)$,

$$
U_{j}=\left(u_{j}^{1}, \ldots, u_{j}^{k}\right)=\|P\| a^{j},
$$

where $a^{j}$ is a norming point for the functional on $V$ associated with $M V_{j}$, i.e.,

$$
\left(M V_{j}\right)(x)=\sum_{i=1}^{k}\left(M V_{j}\right)_{i} x_{i}
$$

Here $x=\sum_{i=1}^{k} x_{i} v^{i}$.

REMARK 1.3 (see e.g. [7]). If $V$ is a symmetric space then $M$ is the identity matrix.

REMARK 1.4. By [3, Th. 1] it is easy to see that if $M$ is invertible and $V_{j} \neq 0$ for $j=1, \ldots, n$, then $\operatorname{crit}(P)=\{1, \ldots, n\}$ for any minimal projection $P$. 
THEOREM 1.5 [8]. Every two-dimensional real Banach space is linearly isometric to a subspace of $L_{1}[-\pi / 2, \pi / 2]$.

THEOREM $1.6[4,6] . L_{1}$ is a maximal overspace for any two-dimensional real symmetric Banach space $V$, which means that

$$
\lambda(V)=\lambda\left(V, L_{1}\right)
$$

II. Technical lemmas. In the above $\|x\|=\sum_{j=1}^{n}\left|V_{j}(x)\right|=1$ determines $S_{V}$ and the following lemma confirms that the "corners" of $S_{V}$ $\left(\operatorname{ext}\left(S_{V}\right)\right)$ are given by those $x$ such that $V_{j}(x)=0 k-1$ times.

LEMma 2.1. Let $V=\operatorname{span}\left[v^{1}, \ldots, v^{k}\right]$ be a $k$-dimensional subspace of $l_{1}^{(n)}$. Then $x=\sum_{i=1}^{k} x_{i} v^{i} \in \operatorname{ext}\left(S_{V}\right)$ if and only if the matrix $W$ consisting of all vectors $V_{j}$ (see (1.9)) orthogonal to $x$ has rank $k-1$ and $\|x\|=1$. We understand that $V_{j}$ is orthogonal to $x$ if

$$
V_{j}(x)=\sum_{i=1}^{k}\left(V_{j}\right)_{i} x_{i}=\sum_{i=1}^{k} v_{j}^{i} x_{i}=0 .
$$

Proof. If $k=1$, the result is obvious. So suppose that $k \geq 2$. Let $x \in \operatorname{ext}\left(S_{V}\right)$. Note that there is $j \in\{1, \ldots, n\}$ such that $x$ is orthogonal to $V_{j}$, i.e., the $j$ th coordinate of $x$ with respect to the canonical basis of $\mathbb{R}^{n}$ is 0 : if not, modifying slightly $x_{1}, \ldots, x_{k}$, we can construct $y, z \in S_{V}$ different from $x$ such that $x=(y+z) / 2$.

Now suppose that $\operatorname{rank}(W)<k-1$ and $k>2$. Put

$$
S=\left\{j \in\{1, \ldots, n\}: x \text { is orthogonal to } V_{j}\right\}
$$

and let $l=\operatorname{card}(S)$. Set $Z=V \cap \bigcap_{j \in S} \operatorname{ker}\left(V_{j}\right)$ (we can consider $Z$ as a subspace of $\left.l_{1}^{(n-l)}\right)$. Since $\operatorname{rank}(W)<k-1$, $\operatorname{dim}(Z) \geq 2$. Since $x \in \operatorname{ext}\left(S_{V}\right)$ and $x \in Z, x \in \operatorname{ext}\left(S_{Z}\right)$. But, by the previous part of the proof, $V_{j}(x)=0$ for some $j \notin S$, contrary to the definition of $W$.

Now take $x \in S_{V}$ and suppose that $\operatorname{rank}(W)=k-1$. If $x \notin \operatorname{ext}\left(S_{V}\right)$, then

$$
x=\left(x^{1}+x^{2}\right) / 2
$$

for some $x^{1}, x^{2} \in S_{V}$ different from $x$. Fix $0<c<1$ and define a norm $\|\cdot\|_{c}$ on $V$ by

$$
\|y\|_{c}=c \sum_{j \in S}\left|V_{j}(y)\right|+\sum_{j \notin S}\left|V_{j}(y)\right|
$$

(see (2.1)). Since $\operatorname{rank}(W)=k-1, x^{1}$ and $x^{2}$ are not perpendicular to all $V_{j}$ for $j \in S$. Hence $\|x\|_{c}=1$ and $\left\|x^{i}\right\|_{c}<1$ for $i=1,2$, contrary to (2.2). 
Corollary 2.2. Let $V=\operatorname{span}\left[v^{1}, v^{2}\right]$ be a 2-dimensional subspace of $l_{1}^{(n)}$. Then $x=x_{1} v^{1}+x_{2} v^{2} \in \operatorname{ext}\left(S_{V}\right)$ if and only if there exists $V_{j} \neq 0$ which is orthogonal to $x$ and $\|x\|_{1}=1$.

Definition 2.3. Given two nonnegative numbers $a, b, a>b$, we denote by $V_{a, b}$ the two-dimensional subspace of $l_{1}^{(4)}$ spanned by

$$
v^{1}=(a, b,-a,-b), \quad v^{2}=(b, a, b, a) .
$$

Let $W_{a, b}$ be the $2 \times 4$ matrix with rows $v^{1}, v^{2}$.

Analogously, let $a=\left(a_{1}, \ldots, a_{n}\right)$ and $b=\left(b_{1}, \ldots, b_{n}\right)$ be two vectors with nonnegative coordinates and with $a_{i}>b_{i}$ for any $i \in\{1, \ldots, n\}$. Let $W_{[a, b]}$ be the $2 \times 4 n$ matrix consisting of $W_{a_{1}, b_{1}}, \ldots, W_{a_{n}, b_{n}}$. Then we denote by $V_{[a, b]}$ the subspace of $l_{1}^{(4 n)}$ spanned by the rows of $W_{[a, b]}$. We write $V_{[a, b]}$ for the space generated by $a, b$. Observe that $V_{[a, b]}$ is a symmetric space with respect to the basis $v^{1}, v^{2}$, where $v^{1}, v^{2}$ denote the rows of $W_{[a, b]}$.

REMARK 2.4. It is a simple consequence of [1, Lemma 1] that each two-dimensional real symmetric space with a polygonal unit ball having $8 n$ extreme points is linearly isometric to $V_{[a, b]}$ for some $a, b \in \mathbb{R}^{n}$ with nonnegative coordinates.

Lemma 2.5. Let $V_{[a, b]} \subset l_{1}^{(4 n)}$ be the space generated by $a, b \in \mathbb{R}_{+}^{n}$. Put

$$
\|(x, y)\|=\left\|x v^{1}+y v^{2}\right\|_{1} .
$$

For $j=1, \ldots, 4$ n define

$$
U_{j}=\left(V_{j} /\left\|V_{j}\right\|\right) \lambda
$$

where

$$
\lambda=\frac{1}{\sum_{i=1}^{n} 2\left(a_{i}^{2}+b_{i}^{2}\right) /\left\|\left(a_{i}, b_{i}\right)\right\|}
$$

and $V_{j}$ are given by (1.9). For $i=1,2$ let $u^{i} \in \mathbb{R}^{4 n}$ be the vector associated with $U_{1}, \ldots, U_{4 n}$ by (1.9). Then the operator $P_{[a, b]}$ defined by

$$
P_{[a, b]} x=u^{1}(x) v^{1}+u^{2}(x) v^{2}
$$

belongs to $\mathcal{P}\left(l_{1}^{(4 n)}, V_{[a, b]}\right)$ and $\left\|P_{[a, b]}\right\|=\lambda$.

Proof. Note that by the definition of $v^{1}$ and $v^{2}, u^{1}\left(v^{2}\right)=u^{2}\left(v^{1}\right)=0$. Observe that by symmetry,

$$
u^{1}\left(v^{1}\right)=u^{2}\left(v^{2}\right)=\lambda \sum_{i=1}^{n} 2\left(a_{i}^{2}+b_{i}^{2}\right) /\left\|\left(a_{i}, b_{i}\right)\right\|=1 .
$$

Hence the orthonormality conditions (1.5) are satisfied and consequently, $P_{[a, b]} \in \mathcal{P}\left(l_{1}^{(4 n)}, V_{[a, b]}\right)$. 
To show that $\left\|P_{[a, b]}\right\|=\lambda$, observe that for any $j \in\{1, \ldots, 4 n\}$,

$$
\begin{aligned}
\left\|P_{[a, b]} e_{j}\right\| & =\left\|\sum_{j=1}^{4 n} u_{j}^{1} v^{1}+u_{j}^{2} v^{2}\right\|_{1} \\
& =\lambda\left\|\left(a_{i}, b_{i}\right) /\right\|\left(a_{i}, b_{i}\right)\|\|=\lambda \quad \text { (by symmetry) }
\end{aligned}
$$

which completes the proof. (Here $i$ is so chosen that $j=4 i-k$, where $k \in\{0,1,2,3\}$.

Lemma 2.6. Let $V_{[a, b]}$ be the space generated by $a, b \in \mathbb{R}^{n}$. Suppose that

$$
0 \leq b_{i}<a_{i}, \quad b_{i} / a_{i}<b_{i+1} / a_{i+1} .
$$

Then

$$
\left\|\left(a_{i}, b_{i}\right)\right\|=2\left(\sum_{j \leq i} a_{j}\left(a_{i}+b_{i}\right)+\sum_{j>i} a_{i}\left(a_{j}+b_{j}\right)\right),
$$

where $\sum_{j>n}=0$ by definition.

Proof. Note that, by $(2.8)$, if $j \leq i$, then

$\left|a_{i} a_{j}+b_{i} b_{j}\right|+\left|-a_{i} a_{j}+b_{i} b_{j}\right|+\left|b_{i} a_{j}+a_{i} b_{j}\right|+\left|-b_{i} a_{j}+a_{i} b_{j}\right|=2 a_{j}\left(a_{i}+b_{i}\right)$.

Hence the result follows from the definition of $v^{1}, v^{2}$ and (2.4).

Now we recall the well known fact that a (weighted) harmonic mean of $n$ nonnegative numbers is no greater than the (weighted) arithmetic mean of these numbers:

Lemma 2.7 Let $a_{1}, \ldots, a_{n} \in \mathbb{R}_{+} \backslash\{0\}$. Then

$$
\frac{1}{\sum_{i=1}^{n} \lambda_{i} a_{i}^{-1}} \leq \sum_{i=1}^{n} \lambda_{i} a_{i}
$$

where $0 \leq \lambda_{i} \leq 1$ and $\sum_{i=1}^{n} \lambda_{i}=1$.

Proof. By cross-multiplying, (2.10) follows easily from the facts that

$$
a_{i} / a_{j}+a_{j} / a_{i} \geq 2 \text { and } 1=\sum_{i=1}^{n} \lambda_{i}^{2}+2 \sum_{j<i} \lambda_{i} \lambda_{j} .
$$

LEMMA 2.8. The following trigonometric identities are satisfied:

$$
\begin{aligned}
& \frac{(\cos 2 a-\sin 2 a) \sin ((a+b) / 2)}{\cos a \cos ((a-b) / 2)} \\
& -\frac{2 \sin a(\cos a+\sin a-\cos b-\sin b)}{\sin (a-b)}=\frac{\sin ((b-3 a) / 2)}{\cos a \cos ((a-b) / 2)}
\end{aligned}
$$


$(2.12)$

$$
\begin{aligned}
& \frac{2(\cos b-\sin b) \sin ((a+b) / 2)}{\cos ((a-b) / 2)} \\
& +\frac{(\cos 2 b-\sin 2 b)(\cos a+\sin a-\cos b-\sin b)}{\sin (a-b)(\cos b+\sin b)} \\
& \quad=\frac{\sin ((a-3 b) / 2)+\cos ((a-3 b) / 2)}{\cos ((a-b) / 2)(\sin b+\cos b)}
\end{aligned}
$$

(2.13) $2 \cos ((b-c) / 2)(\cos b-\sin b)(\sin ((a+b) / 2)$

$-(\cos 2 b-\sin 2 b) \sin ((a-c) / 2)$

$-2 \sin b \cos ((a-b) / 2)(\cos ((b+c) / 2)-\sin ((b+c) / 2))$

$$
=\sin ((a+c-2 b) / 2) ;
$$

$$
\begin{array}{r}
\frac{2 \sin a(\cos b+\sin b)(\sin (b-c)+\sin (c-a)+\sin (a-b))}{\sin (a-b) \sin (b-c)} \\
-\frac{2 \sin a(\cos b+\sin b-\cos c-\sin c)}{\sin (b-c)} \\
=\frac{2 \sin a(\cos b+\sin b-\cos a-\sin a)}{\sin (a-b)}
\end{array}
$$

$$
\begin{array}{r}
\cos ((b-c) / 2) \sin ((a+b) / 2)+\cos b \sin ((c-a) / 2) \\
=\cos ((a-b) / 2) \sin ((b+c) / 2) \\
1=2 \sin \frac{\pi}{8 l} \sum_{j=1}^{l}\left(\cos \frac{\pi(2 j-1)}{8 l}+\sin \frac{\pi(2 j-1)}{8 l}\right)
\end{array}
$$

and

$$
\begin{aligned}
& \sin (b-a)+\sin (a-c)+\sin (c-b) \\
& \quad=-4 \sin ((b-a) / 2) \sin ((c-a) / 2) \sin ((b-c) / 2) .
\end{aligned}
$$

P r o of. Since to prove (2.11)-(2.17) we only use the basic trigonometric formulas and routine calculations, we restrict ourselves to indicating only the main steps.

To show (2.11) observe that

$$
\begin{aligned}
& \frac{(\cos 2 a-\sin 2 a) \sin ((a+b) / 2)}{\cos a \cos ((a-b) / 2)}-\frac{2 \sin a(\cos a+\sin a-\cos b-\sin b)}{\sin (a-b)} \\
& =\frac{(\cos 2 a-\sin 2 a) \sin ((a+b) / 2)}{\cos a \cos ((a-b) / 2)}-\frac{\sin 2 a(\cos ((a+b) / 2)-\sin ((a+b) / 2))}{\cos a \cos ((a-b) / 2)} \\
& =\frac{\sin ((b-3 a) / 2)}{\cos a \cos ((a-b) / 2)} .
\end{aligned}
$$


To show (2.12) observe that

$$
\begin{aligned}
\frac{2(\cos b-\sin b) \sin ((a+b) / 2)}{\cos ((a-b) / 2)} & +\frac{(\cos 2 b-\sin 2 b)(\cos a+\sin a-\cos b-\sin b)}{\sin (a-b)(\cos b+\sin b)} \\
= & \frac{2 \cos 2 b \sin ((a+b) / 2)}{\cos ((a-b) / 2)(\cos b+\sin b)} \\
& +\frac{(\cos 2 b-\sin 2 b)(\cos ((a+b) / 2)-\sin ((a+b) / 2))}{2 \cos ((a-b) / 2)(\cos b+\sin b)} \\
= & \frac{\sin ((a-3 b) / 2)+\cos ((a-3 b) / 2)}{\cos ((a-b) / 2)(\sin b+\cos b)} .
\end{aligned}
$$

To prove (2.13) note that

$$
\begin{aligned}
2 \cos & ((b-c) / 2)(\cos b-\sin b) \sin ((a+b) / 2)-(\cos 2 b-\sin 2 b) \sin ((a-c) / 2) \\
& -2 \sin b \cos ((a-b) / 2)[\cos ((b+c) / 2)-\sin ((b+c) / 2)] \\
=2 \cos ((b-c) / 2)(\cos b-\sin b)[\sin ((a-b) / 2) \cos b+\cos ((a-b) / 2) \sin b] & \\
& -(\cos 2 b-\sin 2 b) \sin ((a-c) / 2) \\
& -2 \sin b \cos ((a-b) / 2)[\cos ((c-b) / 2) \cos b-\sin ((c-b) / 2) \sin b \\
& -\sin ((c-b) / 2) \cos b-\cos ((c-b) / 2) \sin b] \\
= & 2 \cos ^{2} b \cos ((b-c) / 2) \sin ((a-b) / 2) \\
& -2 \sin ^{2} b \cos ((b-c) / 2) \sin ((a-b) / 2) \\
& +\sin 2 b[\cos ((b-c) / 2) \cos ((a-b) / 2)-\cos ((b-c) / 2) \sin ((a-b) / 2)] \\
& -(\cos 2 b-\sin 2 b) \sin ((a-c) / 2) \\
& -2 \sin ^{2} b \cos ((a-b) / 2)[-\sin ((c-b) / 2)-\cos ((c-b) / 2)] \\
& -\sin ^{2} b \cos ((a-b) / 2)[\cos ((c-b) / 2)-\sin ((c-b) / 2)] \\
=2 & \cos ^{2} b \cos ((b-c) / 2) \sin ((a-b) / 2)-\cos 2 b \sin ((a-c) / 2) \\
& -2 \sin ^{2} b \cos ((a-b) / 2) \sin ((b-c) / 2) \\
=2 & \left(\cos ^{2} b-\sin { }^{2} b\right)[\cos ((b-c) / 2) \sin ((a-b) / 2)-\cos 2 b \sin ((a-c) / 2)] \\
& \left.+\left(\sin ^{2} b-\cos { }^{2} b\right) \sin [(a-b) / 2+(b-c) / 2)\right] \\
= & \cos ^{2} b \cos ((b-c) / 2) \sin ((a-b) / 2)-\sin ^{2} b \cos ((a-b) / 2) \sin ((b-c) / 2) \\
& +\sin ^{2} b \cos ((b-c) / 2) \sin ((a-b) / 2) \\
& -\cos ^{2} b \cos ((a-b) / 2) \sin ((b-c) / 2) \\
= & \cos ^{2} b[\cos ((b-c) / 2) \sin ((a-b) / 2)-\cos ((a-b) / 2) \sin ((b-c) / 2)] \\
& +\sin ^{2} b[-\cos ((a-b) / 2) \sin ((b-c) / 2)+\cos ((b-c) / 2) \sin ((a-b) / 2)] \\
= & \sin ((a+c-2 b) / 2) . \\
&
\end{aligned}
$$


To show (2.14), note that

$$
\begin{aligned}
& \frac{2 \sin a(\cos b+\sin b)(\sin (b-c)+\sin (c-a)+\sin (a-b))}{\sin (a-b) \sin (b-c)} \\
& -\frac{2 \sin a(\cos b+\sin b-\cos c-\sin c)}{\sin (b-c)} \\
& =\frac{2 \sin a(\cos b+\sin b-\sin a-\cos a)}{\sin (a-b)} \\
& +\frac{2 \sin a \sin (b-c)(\cos a+\sin a)}{\sin (a-b) \sin (b-c)} \\
& +\frac{2 \sin a(\cos b+\sin b) \sin (c-a)}{\sin (a-b) \sin (b-c)}+\frac{2 \sin a(\cos c+\sin c)}{\sin (b-c)} .
\end{aligned}
$$

To finish the proof we have to show that the sum of the second and third terms from the above formula is

$$
-\frac{2 \sin a(\cos c+\sin c)}{\sin (b-c)} .
$$

But this follows immediately from the fact that

$\sin (b-c)(\cos a+\sin a)+\sin (c-a)(\cos b+\sin b)=\sin (b-a)(\cos c+\sin c)$.

To show (2.15) note that

$$
\begin{aligned}
\cos ((b-c) / 2) \sin ((a+b) / 2)+\cos b \sin ((c-a) / 2) \\
\quad=\cos ((b+c) / 2-c) \sin ((a+b) / 2)+\cos b \sin ((b+c) / 2-(a+b) / 2) \\
=\sin ((b+c) / 2)(\cos b \cos ((a+b) / 2)+\sin b \sin ((a+b) / 2)) \\
=\cos ((a-b) / 2) \sin ((b+c) / 2) .
\end{aligned}
$$

To prove (2.16), observe that for odd $l$,

$$
\begin{aligned}
2 \sin \frac{\pi}{8 l} \sum_{j=1}^{l}\left(\cos \frac{\pi(2 j-1)}{8 l}+\sin \frac{\pi(2 j-1)}{8 l}\right) \\
=4 \sin \frac{\pi}{8 l}\left(\cos \frac{\pi}{8}+\sin \frac{\pi}{8}\right)\left(\frac{1}{2}+\sum_{j=1}^{(l-1) / 2} \cos \frac{\pi j}{4 l}\right) \\
=2 \sin \frac{\pi}{8 l}\left(\cos \frac{\pi}{8}+\sin \frac{\pi}{8}\right) \frac{\sin \frac{((l-1) / 2+1 / 2) \pi}{4 l}}{\sin \frac{\pi}{8 l}} \\
=2 \sin \frac{\pi}{8}\left(\cos \frac{\pi}{8}+\sin \frac{\pi}{8}\right)=1 .
\end{aligned}
$$


If $l$ is even then

$$
\begin{aligned}
& 2 \sin \frac{\pi}{8 l} \sum_{j=1}^{l}\left(\cos \frac{\pi(2 j-1)}{8 l}+\sin \frac{\pi(2 j-1)}{8 l}\right) \\
& =2 \sin \frac{\pi}{4 l} \sum_{j=1}^{l / 2}\left(\cos \frac{\pi(2 j-1)}{4 l}+\sin \frac{\pi(2 j-1)}{4 l}\right)
\end{aligned}
$$

which reduces the proof to the previous case.

The proof of (2.17) is an easy calculation, so we omit it.

LEMMA 2.9. Let $A_{n}=\left(a_{i j}\right)_{i, j=1}^{n}$ be an $n \times n$ symmetric matrix given by $a_{i j}=\cos d_{i}\left(\cos d_{j}+\sin d_{j}\right)$ for $1 \leq i \leq j$. Let $A_{n}^{i}$ be the matrix obtained from $A_{n}$ by replacing the ith column by $(1, \ldots, 1)$. Then for any $d_{1}, \ldots, d_{n} \in$ $(0, \pi / 2)$

$$
\begin{aligned}
\operatorname{det}\left(A_{n}\right)= & \cos d_{1}\left(\cos d_{n}+\sin d_{n}\right) \prod_{j=1}^{n-1} \sin \left(d_{j}-d_{j+1}\right) \\
\operatorname{det}\left(A_{n}^{1}\right)= & \left(\cos d_{n}+\sin d_{n}\right) \prod_{j=2}^{n-1} \sin \left(d_{j}-d_{j+1}\right)\left(\cos d_{2}-\cos d_{1}\right) \\
\operatorname{det}\left(A_{n}^{n}\right)= & \cos d_{1}\left(\cos d_{n-1}+\sin d_{n-1}-\cos d_{n}-\sin d_{n}\right) \\
& \times \prod_{j=1}^{n-2} \sin \left(d_{j}-d_{j+1}\right)
\end{aligned}
$$$$
\operatorname{det}\left(A_{n}^{i}\right)=-4 \cos d_{1}\left(\cos d_{n}+\sin d_{n}\right) \prod_{j=1}^{i-2} \sin \left(d_{j}-d_{j+1}\right)
$$

$$
\begin{aligned}
& \times \prod_{j=i+1}^{n-1} \sin \left(d_{j}-d_{j+1}\right) \sin \left(\left(d_{i-1}-d_{i}\right) / 2\right) \\
& \times \sin \left(\left(d_{i-1}-d_{i+1}\right) / 2\right) \sin \left(\left(d_{i}-d_{i+1}\right) / 2\right),
\end{aligned}
$$

for $i=2, \ldots, n-1$. Here $\prod_{i=l}^{j}=1$ if $l>j$, by definition.

Proof. We prove the lemma by induction on $n$. If $n=2$, then easy calculations show that (2.18)-(2.20) hold true. To prove (2.18) for any $n \in \mathbb{N}$, add the second column of $A_{n}$ multiplied by $-\cos d_{1} / \cos d_{2}$ to the first one, apply the induction hypothesis to the $(n-1) \times(n-1)$ matrix given by $d_{2}, \ldots, d_{n}$ and the standard formula for calculating determinants.

To show (2.19) for any $n \in \mathbb{N}$, add the second column of $A_{n}^{1}$ multiplied by $-\cos d_{1} / \cos d_{2}$ to the first one and proceed as in the previous case. The same reasoning applies to $(2.20)$. To show (2.21), we first consider the case 
$n=3$. Note that

$$
\begin{aligned}
\operatorname{det}\left(A_{3}^{2}\right)= & \cos d_{1}\left(\cos d_{3}+\sin d_{3}\right) \\
& \times\left(\sin \left(d_{2}-d_{1}\right)+\sin \left(d_{1}-d_{3}\right)+\sin \left(d_{3}-d_{2}\right)\right) \\
= & -4 \cos d_{1}\left(\cos d_{3}+\sin d_{3}\right) \sin \left(\left(d_{1}-d_{2}\right) / 2\right) \\
& \left.\times \sin \left(\left(d_{1}-d_{3}\right) / 2\right) \sin \left(d_{2}-d_{3}\right) / 2\right) \quad(\text { by }(2.17)),
\end{aligned}
$$

as required.

To prove (2.21) for $n \geq 4$, for $i=3, \ldots, n-1$ add the second column of $A_{n}^{i}$ multiplied by $-\cos d_{1} / \cos d_{2}$ to the first one, and apply the induction hypothesis for $d_{2}, \ldots, d_{n}$ and $i-1$. For $i=2$, add the $(n-1)$ th column of $A_{n}^{i}$ multiplied by $-\left(\cos d_{n}+\sin d_{n}\right) /\left(\cos d_{n-1}+\sin d_{n-1}\right)$ and apply the induction hypothesis for $d_{1}, \ldots, d_{n-1}$ and $i$.

We also need the following obvious fact:

REMARK 2.10. Let $f, g$ be real-valued functions defined on a set $A$, with $f \leq g$. If $g(x)=\max _{y \in A} g(y)$ and $g(x)=f(x)$, then $f(x)=\max _{y \in A} f(y)$.

\section{The main results}

THEOREM 3.1. Let $V$ be a two-dimensional real symmetric space with unit ball having exactly $8 n$ extreme points. Then

$$
\lambda(V) \leq \lambda\left(V_{n}\right),
$$

where $V_{n}$ is the space whose unit ball is a regular polyhedron having exactly $8 n$ vertices. Moreover, if $\lambda(V)=\lambda\left(V_{n}\right)$, then $V$ is linearly isometric to $V_{n}$.

Proof. First we consider the case $V=V_{[a, b]}$, where $a=\left(a_{1}, \ldots, a_{n}\right)$, $b=\left(b_{1}, \ldots, b_{n}\right) \in \mathbb{R}^{n}, 0<b_{i}<a_{i}, b_{i} / a_{i}<b_{i+1} / a_{i+1}$, for $i=1, \ldots, n-1$. For $i=1, \ldots, n$, put $r_{i}=\sqrt{a_{i}^{2}+b_{i}^{2}}$, and let $d_{i} \in(0, \pi / 4)$ be so chosen that $a_{i}=r_{i} \cos d_{i}$ and $b_{i}=r_{i} \sin d_{i}$. Without loss, dividing $a_{i}$ and $b_{i}$ by a constant, we can assume that

$$
\sum_{i=1}^{n} r_{i}=1
$$

Put $r=\left(r_{1}, \ldots, r_{n}\right), d=\left(d_{1}, \ldots, d_{n}\right)$ and let

$$
g_{n}(d, r)=\sum_{i=1}^{n} r_{i} \sum_{j=1}^{n} r_{j} a_{i j}(d),
$$

where $a_{i j}(d)=\cos d_{i}\left(\cos d_{j}+\sin d_{j}\right)$ for $i \leq j$ and $a_{i j}=a_{j i}$ for $i>j$. Define

$$
D_{n}=\left\{(d, r) \in \mathbb{R}^{2 n}: 0 \leq r_{i}, \sum_{i=1}^{n} r_{i}=1,0 \leq d_{1} \leq \ldots \leq d_{n} \leq \pi / 4\right\} .
$$


First we will show that for any $n \geq 2, \max _{(d, r) \in D_{n}} g_{n}(d, r)$ is achieved for $r=(1 / n, \ldots, 1 / n)$ and $d=\left(\frac{\pi}{8 n}, \frac{3 \pi}{8 n}, \ldots, \frac{(2 n-1) \pi}{8 n}\right)$. To do this, we apply the Lagrange multiplier method. Consider in $\operatorname{int}\left(D_{n}\right)$ the function

$$
w_{n}(d, r)=g_{n}(d, r)+\lambda\left(1-\sum_{i=1}^{n} r_{i}\right)
$$

Note that

$$
\frac{\partial w_{n}}{\partial r_{i}}(d, r)=2 \sum_{i=1}^{n} a_{i j}(d) r_{j}-\lambda
$$

for $i=1, \ldots, n$. By Cramer's rule and Lemma 2.9, the system of equations

$$
\frac{\partial w_{n}}{\partial r_{i}}(d, r)=2 \sum_{i=1}^{n} a_{i j}(d) r_{j}-\lambda=0
$$

for $i=1, \ldots, n$ has, in $\operatorname{int}\left(D_{n}\right)$, for fixed $\lambda$ and $d$, the solution $r(d, \lambda)=$ $\left(r_{1}(d, \lambda), \ldots, r_{n}(d, \lambda)\right)$ given by

$$
\begin{aligned}
r_{1}(d, \lambda) & =\frac{\lambda\left(\cos d_{2}-\cos d_{1}\right)}{2 \cos d_{1} \sin \left(d_{1}-d_{2}\right)}, \\
r_{i}(d, \lambda) & =\frac{\lambda \sin \left(\left(d_{i+1}-d_{i-1}\right) / 2\right)}{2 \cos \left(\left(d_{i-1}-d_{i}\right) / 2\right) \cos \left(\left(d_{i}-d_{i+1}\right) / 2\right)}, \quad i=2, \ldots, n-1, \\
r_{n}(d, \lambda) & =\frac{-\lambda}{2 \sin \left(d_{n-1}-d_{n}\right)}+\frac{\lambda\left(\cos d_{n-1}+\sin d_{n-1}\right)}{2 \sin \left(d_{n-1}-d_{n}\right)\left(\cos d_{n}+\sin d_{n}\right)} .
\end{aligned}
$$

Now we prove by induction on $n$ that the system of equations

$$
\frac{\partial w_{n}}{\partial d_{i}}(d, r(d, \lambda))=0, \quad i=1, \ldots, n,
$$

reduces to the system

$$
\begin{aligned}
& \sin \left(\left(3 d_{1}-d_{2}\right) / 2\right)=0, \\
& \sin \left(\left(d_{i-1}+d_{i+1}-2 d_{i}\right) / 2\right)=0, \quad i=2, \ldots, n-1, \\
& \cos \left(\left(d_{n-1}-3 d_{n}\right) / 2\right)+\sin \left(\left(d_{n-1}-3 d_{n}\right) / 2\right)=0 .
\end{aligned}
$$

Note that for $n=2$,

$$
\begin{aligned}
\frac{\partial w_{2}}{\partial d_{1}}(d, r(d, \lambda))= & \lambda^{2} r_{1}(d, \lambda)\left(\frac{\left(\cos 2 d_{1}-\sin 2 d_{1}\right) \sin \left(\left(d_{1}+d_{2}\right) / 2\right)}{\cos d_{1} \cos \left(\left(d_{1}-d_{2}\right) / 2\right)}\right. \\
& \left.-\frac{2 \sin d_{1}\left(\cos d_{1}+\sin d_{1}-\cos d_{2}-\sin d_{2}\right)}{\sin \left(d_{1}-d_{2}\right)}\right) \\
= & \lambda^{2} r_{1}(d, \lambda) \frac{\sin \left(\left(d_{2}-3 d_{1}\right) / 2\right)}{\cos d_{1} \cos \left(\left(d_{1}-d_{2}\right) / 2\right)} \quad(\text { by }(2.11))
\end{aligned}
$$


Analogously,

$$
\begin{aligned}
& \frac{\partial w_{2}}{\partial d_{2}}(d, r(d, \lambda)) \\
& =\lambda^{2} r_{2}(d, \lambda)\left(\frac{2\left(\cos d_{2}-\sin d_{2}\right) \sin \left(\left(d_{1}+d_{2}\right) / 2\right)}{\cos \left(\left(d_{1}-d_{2}\right) / 2\right)}\right. \\
& \left.\quad+\frac{\left(\cos 2 d_{2}-\sin 2 d_{2}\right)\left(\cos d_{1}+\sin d_{1}-\cos d_{2}-\sin d_{2}\right)}{\sin \left(d_{1}-d_{2}\right)\left(\cos d_{2}+\sin d_{2}\right)}\right) \\
& \left.=\lambda^{2} r_{2}(d, \lambda) \frac{\sin \left(\left(d_{1}-3 d_{2}\right) / 2\right)+\cos \left(\left(d_{1}-3 d_{2}\right) / 2\right)}{\cos \left(\left(d_{1}-d_{2}\right) / 2\right)\left(\sin d_{2}+\cos d_{2}\right)} \quad \text { (by }(2.12)\right),
\end{aligned}
$$

which proves our claim for $n=2$.

Now for illustration consider first the case $n=3$. Note that

$$
\begin{aligned}
\frac{\partial w_{3}}{\partial d_{1}}(d, r(d, \lambda))= & (2 \lambda)^{2} r_{1}(d, \lambda)\left[\left(\cos 2 d_{1}-\sin 2 d_{1}\right) r_{1}(d, \lambda)\right. \\
& -2 r_{2}(d, \lambda) \sin d_{1}\left(\cos d_{2}+\sin d_{2}\right) \\
& \left.-2 r_{3}(d, \lambda) \sin d_{1}\left(\cos d_{3}+\sin d_{3}\right)\right]
\end{aligned}
$$

By (3.7)-(3.9), (2.17) and (2.14) applied to $d_{1}, d_{2}, d_{3}$ and the second and third terms of the above equality, we get

$$
\frac{\partial w_{3}}{\partial d_{1}}(d, r(d, \lambda))=\frac{\partial w_{2}}{\partial d_{1}}\left(d_{1}, d_{2}, r\left(d_{1}, d_{2}, \lambda\right)\right),
$$

which proves the result for $\frac{\partial w_{3}}{\partial d_{1}}(d, r(d, \lambda))$.

Observe that by (3.7)-(3.9),

$$
\begin{aligned}
\frac{\partial w_{3}}{\partial d_{2}}(d, r(d, \lambda)) & \\
= & \lambda^{2} r_{2}(d, \lambda)\left(\frac{2 \cos \left(\left(d_{2}-d_{3}\right) / 2\right)\left(\cos d_{2}-\sin d_{2}\right) \sin \left(\left(d_{1}+d_{2}\right) / 2\right)}{\cos \left(\left(d_{1}-d_{2}\right) / 2\right) \cos \left(\left(d_{2}-d_{3}\right) / 2\right)}\right. \\
& -\frac{\left(\cos 2 d_{2}-\sin 2 d_{2}\right) \sin \left(\left(d_{1}-d_{3}\right) / 2\right)}{\cos \left(\left(d_{1}-d_{2}\right) / 2\right) \cos \left(\left(d_{2}-d_{3}\right) / 2\right)} \\
& \left.-\frac{2 \sin d_{2}\left[\cos \left(\left(d_{2}+d_{3}\right) / 2\right)-\sin \left(\left(d_{2}+d_{3}\right) / 2\right)\right] \cos \left(\left(d_{1}-d_{2}\right) / 2\right)}{\cos \left(\left(d_{1}-d_{2}\right) / 2\right) \cos \left(\left(d_{2}-d_{3}\right) / 2\right)}\right) \\
= & \lambda^{2} r_{2}(d, \lambda) \frac{\sin \left(\left(d_{1}+d_{3}-2 d_{2}\right) / 2\right)}{\cos \left(\left(d_{1}-d_{2}\right) / 2\right) \cos \left(\left(d_{2}-d_{3}\right) / 2\right)} \quad(\text { by }(2.13)),
\end{aligned}
$$

which proves our claim. 
Note that

$$
\begin{aligned}
\frac{\partial w_{3}}{\partial d_{3}}(d, r(d, \lambda))= & (2 \lambda)^{2} r_{3}(d, \lambda)\left[2 \cos d_{1}\left(\cos d_{3}-\sin d_{3}\right) r_{1}(d, \lambda)\right. \\
& +2 r_{2}(d, \lambda) \cos d_{2}\left(\cos d_{3}-\sin d_{3}\right) \\
& \left.+r_{3}(d, \lambda)\left(\cos 2 d_{3}-\sin 2 d_{3}\right)\right]
\end{aligned}
$$

By (3.7)-(3.9), (2.17) and (2.15) applied to $d_{1}, d_{2}, d_{3}$ and the first and second terms of the above equality, we get

$$
\frac{\partial w_{3}}{\partial d_{3}}(d, r(d, \lambda))=\frac{\partial w_{2}}{\partial d_{3}}\left(d_{2}, d_{3}, r\left(d_{2}, d_{3}, \lambda\right)\right),
$$

which proves the result for $\frac{\partial w_{3}}{\partial d_{3}}(d, r(d, \lambda))$.

Now fix $n \in \mathbb{N}, n \geq 4$. Observe that

$$
\begin{aligned}
\frac{\partial w_{n}}{\partial d_{i}}(d, r(d, \lambda))= & 4 \lambda^{2} r_{i}(d, \lambda)\left(\sum_{j=1}^{i-1} 2 r_{j}(d, \lambda) \cos d_{j}\left(\cos d_{i}-\sin d_{i}\right)\right. \\
& +r_{i}(d, \lambda)\left(\cos 2 d_{i}-\sin 2 d_{i}\right) \\
& \left.-\sum_{j>i} r_{j}(d, \lambda) 2 \sin d_{i}\left(\cos d_{j}+\sin d_{j}\right)\right)
\end{aligned}
$$

Hence to prove (3.11) and (3.12) for $i=2, \ldots, n-2$, apply (2.14) to $d_{n-1}$ and $d_{n}$ in the last two terms of the sum in (3.14) and the induction hypothesis for $n-1$ and $d_{1}, \ldots, d_{n-1}$. To prove (3.12) for $i=n-1$ and (3.13), apply (2.15) to $d_{1}, d_{2}$ in the first and second terms of $\sum_{j=1}^{i-1} 2 r_{j}(d, \lambda) \cos d_{j}\left(\cos d_{i}-\sin d_{i}\right)$ and the induction hypothesis for $n-1$ and $d_{2}, \ldots, d_{n}$.

Now note that, since we consider $d_{1}, \ldots, d_{n}$ belonging to $(0, \pi / 4)$, by (3.7)-(3.13) the system of equations

$$
\frac{\partial w_{n}}{\partial r_{i}}(d, r(d, \lambda))=0, \quad \frac{\partial w_{n}}{\partial d_{i}}(d, r(d, \lambda))=0, \quad \sum_{i=1}^{n} r_{i}=1,
$$

has for $n=1,2, \ldots$ in $\operatorname{int}\left(D_{n}\right)$ the only solution

$$
d^{0}=\left(\frac{\pi}{8 n}, \ldots, \frac{(2 n-1) \pi}{8 n}\right), \quad r^{0}=\left(\frac{1}{n}, \ldots, \frac{1}{n}\right) .
$$

Hence if we prove that the function $g_{n}$ does not attain a global maximum on the boundary of $D_{n}$ then it has to attain it at $\left(d^{0}, r^{0}\right)$. This will also be shown by induction on $n$. If $n=1$, then $g_{1}(d)=\cos d(\cos d+\sin d)$. Hence $g_{1}^{\prime \prime}(d)=-2(\cos 2 d+\sin 2 d)$, which shows that $g_{1}$ attains a global maximum at $d^{0}=\pi / 8$. Now take any $n \geq 2$. Since $D_{n}$ is a compact set, $g_{n}$ attains a global maximum at some point $(d, r) \in D_{n}$. If $r_{i}>0$ and $d_{i}<d_{i+1}$ for $i=1, \ldots, n-1$, and $d_{1}=0$ or $d_{n}=\pi / 4$, then, by easy calculations, 
$g_{n}(d, r)=\max \left\{g_{n}(w, z):(w, z) \in D_{n}^{\varepsilon}\right\}$, where

$$
D_{n}^{\varepsilon}=\left\{(d, r) \in \mathbb{R}^{2 n}: r_{i}>0, \sum_{i} r_{i}=1,-\varepsilon<d_{1}<\ldots<d_{n}<\pi / 4+\varepsilon\right\}
$$

and $0<\varepsilon<\min \left\{d_{2}-d_{1}, d_{n}-d_{n-1}\right\}$. But $D_{n}^{\varepsilon}$ is an open set and by (3.7)(3.13) the maximum on this set can be attained at $\left(d^{0}, r^{0}\right)$ given by (3.15); a contradiction. So suppose that $d_{i}=d_{i+1}$ for some $i \in\{1, \ldots, n-1\}$. Then

$$
\begin{aligned}
g_{n}(d, r) & =g_{n-1}\left(d_{1}, \ldots, d_{i-1}, d_{i+1}, \ldots, d_{n}, r_{1}, \ldots, r_{i-1}, r_{i}+r_{i+1}, \ldots, r_{n}\right) \\
& <g_{n-1}\left(\frac{\pi}{8(n-1)}, \ldots, \frac{(2 n-3) \pi}{8(n-1)}, \frac{1}{n-1}, \ldots, \frac{1}{n-1}\right) \\
& =\cos \frac{\pi}{8(n-1)} \cdot \frac{\sum_{j=1}^{n-1}\left(\cos \frac{(2 j-1) \pi}{8(n-1)}+\sin \frac{(2 j-1)}{8(n-1)}\right)}{n-1} \\
& =\frac{\cos \frac{\pi}{8(n-1)}}{2(n-1) \sin \frac{\pi}{8(n-1)}} \quad(\text { by }(2.16)) .
\end{aligned}
$$

Note that the function $f(x)=(4 / x) \cos (\pi / x) / \sin (\pi / x)$ is strictly increasing for $x>0$. Hence

$$
\left.\frac{\cos \frac{\pi}{8(n-1)}}{2(n-1) \sin \frac{\pi}{8(n-1)}}<\frac{\cos \frac{\pi}{8 n}}{2 n \sin \frac{\pi}{8 n}}=g_{n}\left(d^{0}, r^{0}\right) \quad \text { (by }(2.16)\right),
$$

which shows that $g_{n}$ does not attain its maximum on the boundary of $D_{n}$. The same reasoning applies to the case $r_{i}=0$ for some $i \in\{1, \ldots, n\}$. Hence

$$
\max _{(d, r) \in D_{n}} g_{n}(d, r)=g_{n}\left(d^{0}, r^{0}\right),
$$

as required.

Observe that the unit ball of the space generated by $\left(d^{0}, r^{0}\right)$ is a regular $8 n$-polygon, so this space is isometric to $V_{n}$. To finish the proof of our theorem we apply the idea given in Remark 2.10 twice. Note that by Lemma 2.7 ,

$$
g_{n}(d, r) \geq f_{n}(d, r)=\left(\sum_{i=1}^{n} \frac{r_{i}}{\sum_{j=1}^{n} r_{j} a_{i j}(d)}\right)^{-1} .
$$

By Lemma $2.5, f_{n}(d, r)$ is the norm of the projection $P_{[a, b]}$ defined in Lemma 2.5 (we use polar coordinates). Note that, by (3.6),

$$
f_{n}\left(d^{0}, r^{0}\right)=g_{n}\left(d^{0}, r^{0}\right) .
$$

Now we show that the projection $P_{[a, b]}$ associated with $\left(d^{0}, r^{0}\right)$ is a minimal projection. To do this, by Theorem 1.2, Remarks 1.3 and 1.4, we have to show that for $i=1, \ldots, n$,

$$
\left(a_{i}, b_{i}\right) /\left\|\left(a_{i}, b_{i}\right)\right\|=\left(r_{i}^{0} \cos d_{i}^{0}, r_{i}^{0} \sin d_{i}^{0}\right) /\left\|\left(r_{i}^{0} \cos d_{i}^{0}, r_{i}^{0} \sin d_{i}^{0}\right)\right\|
$$


is the only norming point for the functional associated with $\left(a_{i}, b_{i}\right)$ (see Th. 1.2). But by Lemma 2.6,

$$
\left\|\left(a_{i}, b_{i}\right)\right\|=\sum_{j=1}^{n} r_{j}^{0} a_{i j}\left(d^{0}\right) .
$$

By (3.6) and Corollary 2.2, all the extreme points of the unit ball of $V_{[a, b]}$ lie on the same Euclidean sphere. This shows that $\left(a_{i}, b_{i}\right) /\left\|\left(a_{i}, b_{i}\right)\right\|$ is the only norming point for the functional $\left(a_{i}, b_{i}\right)$. To finish the proof of the theorem, note that, by Theorems 1.5 and 1.6 and Remark 2.4, for any two-dimensional real symmetric Banach space with $8 n$ extreme points,

$$
\lambda(V)=\lambda\left(V_{[a, b]}\right)=\lambda\left(V_{[a, b]}, l^{1}\right) \leq\left\|P_{[a, b]}\right\| \leq \lambda\left(V_{n}, l^{1}\right)=\lambda\left(V_{n}\right) .
$$

Moreover, by Remark 2.4 and the above reasoning, if $\lambda(V)=\lambda\left(V_{n}\right)$, then $V$ has to be linearly isometric to $V_{n}$.

The proof of Theorem 3.1 is complete.

Now we apply Theorem 3.1 to reprove in a simple way a result of [1] and [5] concerning arbitrary two-dimensional real symmetric spaces.

TheOREM $3.2[1,5]$. For any two-dimensional symmetric real Banach space $V$,

$$
\lambda(V) \leq \lambda\left(l_{2}^{(2)}\right)
$$

Proof. By Theorem 1.6, we can assume that $V \subset L_{1}[-\pi / 2, \pi / 2]$. Hence we can approximate $V$, in the sense of the Banach-Mazur distance, by subspaces $V_{\left[a^{n}, b^{n}\right]} \subset l^{1}$ whose unit balls have exactly $8 n$ extreme points. Since the function $V \mapsto \lambda(V)$ is continuous with respect to the Banach-Mazur distance, by Theorems 1.5 and 1.6,

$$
\begin{aligned}
\lambda(V) & =\lim _{n} \lambda\left(V_{\left[a^{n}, b^{n}\right]}\right) \leq \lim _{n} \lambda\left(V_{n}\right) \\
& =\lim _{n} \frac{\cos \frac{\pi}{8 n}}{2 n \sin \frac{\pi}{8 n}} \quad(\operatorname{by}(2.17)) \\
& =4 / \pi=\lambda\left(l_{2}^{(2)}\right),
\end{aligned}
$$

as required.

\section{References}

[1] B. L. Chalmers, C. Franchetti and M. Giaquinta, On the self-length of twodimensional Banach spaces, Bull. Austral. Math. Soc. 53 (1996), 101-107.

[2] B. L. Chalmers and F. T. Metcalf, The determination of minimal projections and extensions in $L^{1}$, Trans. Amer. Math. Soc. 329 (1992), 289-305.

[3] -, - A characterization and equations for minimal projections and extensions, J. Operator Theory 32 (1994), 31-46. 
[4] B. L. Chalmers and F. T. Metcalf, A simple formula showing $L^{1}$ is a maximal overspace for two-dimensional real spaces, Ann. Polon. Math. 56 (1992), 303-309.

[5] H. Koenig, Projections onto symmetric spaces, Quaestiones Math. 18 (1995), 199 220.

[6] J. Lindenstrauss, On the extension of operators with a finite-dimensional range, Illinois J. Math. 8 (1964), 488-499.

[7] N. Tomczak-Jaegermann, Banach-Mazur Distances and Finite-Dimensional Operator Ideals, Wiley, New York, 1989.

[8] D. Yost, $L_{1}$ contains every two-dimensional normed space, Ann. Polon. Math. 49 (1988), 17-19.

Department of Mathematics

University of California

Riverside, CA, 92521, U.S.A.

E-mail: blc@math.ucr.edu
Institute of Mathematics

Jagiellonian University Reymonta 4

30-059 Kraków, Poland

E-mail: lewicki@im.uj.edu.pl

Reçu par la Rédaction le 2.12.1998

Révisé le 27.10.1999 